\title{
Bevacizumab Plus Erlotinib Combination Therapy for Advanced Hereditary Leiomyomatosis and Renal Cell Carcinoma-Associated Renal Cell Carcinoma: A Multicenter Retrospective Analysis in Korean Patients
}

\author{
Yeonjoo Choi, $\mathrm{MD}^{1}$ \\ Bhumsuk Keam, MD, PhD ${ }^{1}$ \\ Miso Kim, MD' \\ Shinkyo Yoon, MD2 \\ Dalyong Kim, $\mathrm{MD}^{3}$ \\ Jong Gwon Choi, MD, $\mathrm{PhD}^{4}$ \\ Ja Young Seo, MD \\ Inkeun Park, MD, PhD \\ Jae Lyun Lee, MD, PhD²
}

\begin{abstract}
${ }^{1}$ Division of Medical Oncology, Department of Internal Medicine, Seoul National University Hospital, Seoul, ${ }^{2}$ Department of Oncology, Asan Medical Center, University of Ulsan College of Medicine, Seoul, ${ }^{3}$ Division of

Hematology and Medical Oncology,

Dongguk University Ilsan Hospital, Goyang,

${ }^{4}$ Department of Internal Medicine,

Konyang University Hospital, Daejeon,

${ }^{5}$ Department of Laboratory Medicine and

${ }^{6}$ Division of Medical Oncology,

Department of Internal Medicine, Gachon

University Gil Medical Center, Incheon, Korea
\end{abstract}

Correspondence: Inkeun Park, MD, PhD

Division of Medical Oncology, Department of

Internal Medicine, Gachon University

Gil Medical Center, 21 Namdong-daero

774beon-gil, Namdong-gu, Incheon 21565, Korea

Tel: 82-32-460-3229

Fax: 82-32-460-2391

E-mail: ingni79@gilhospital.com

Received February 10, 2019

Accepted March 23, 2019

Published Online March 25, 2019

\begin{abstract}
Purpose
Hereditary leiomyomatosis and renal cell carcinoma (HLRCC) is a rare genetic syndrome resulting from germline mutations in fumarate hydratase. The combination of bevacizumab plus erlotinib showed promising interim results for HLRCC-associated renal cell carcinoma (RCC). Based on these results, we analyzed the outcome of bevacizumab plus erlotinib in Korean patients with HLRCC-associated RCC.
\end{abstract}

\section{Materials and Methods}

We retrospectively reviewed the efficacy and safety of bevacizumab plus erlotinib in patients with HLRCC-associated RCC who were confirmed to have germline mutations in fumarate hydratase. The primary endpoint was the objective response rate (ORR), while the secondary endpoints were progression-free survival (PFS) and overall survival (OS).

\section{Results}

We identified 10 patients with advanced HLRCC-associated RCC who received bevacizumab plus erlotinib. Median age at diagnosis was 41 years, and five of the patients had received the combination as first- or second-line treatments. The ORR was 50\% and the median PFS and OS were 13.3 and 14.1 months, respectively. Most adverse events were predictable and manageable by conventional measures, except for one instance where a patient died of gastrointestinal bleeding.

\section{Conclusion}

This is the first real-world outcome of the treatment of advanced HLRCC-associated RCC. Bevacizumab plus erlotinib therapy showed promising activity with moderate toxicity. We should be increasingly aware of HLRCC-associated RCC and bevacizumab plus erlotinib should be a first-line treatment for this condition, unless other promising data are published.

\section{Key words}

Hereditary leiomyomatosis and renal cell carcinoma, Bevacizumab, Erlotinib, Renal cell carcinoma, Fumarate hydratase, Non-clear cell

\section{Introduction}

Hereditary leiomyomatosis and renal cell carcinoma (HLR$\mathrm{CC}$ ) syndrome is a genetic syndrome resulting from germline mutations in fumarate hydratase (FH) [1]. This autosomal dominant condition is characterized by cutaneous leiomy- omas, early-onset multiple uterine leiomyomas, and an aggressive form of type 2 papillary renal cell carcinoma (RCC) [2,3]. Although RCC arising in HLRCC syndrome has been described as type 2 papillary RCC, the recently updated World Health Organization (WHO) 2016 genitourinary cancer classification added RCC in HLRCC as a new entity, HLRCC-associated RCC [4]. 
More than 180 families with FH germline mutations have been reported [3] but the exact incidence of HLRCC syndrome or HLRCC-associated RCC remains unknown as this entity has had a low awareness among urologists, medical oncologists, and pathologists until today. A considerable proportion of patients with HLRCC-associated RCC might have been diagnosed and treated as having sporadic papillary RCC and even after correct diagnosis, there has been no specific treatment option for advanced HLRCC-associated RCC. The most commonly used treatment might be mammalian target of rapamycin (mTOR) inhibitors and vascular endothelial growth factor receptor tyrosine kinase inhibitors (VEGFR TKIs), similar to the treatment of non-clear cell RCC [5].

With the accumulation of pathobiological knowledge underlying HLRCC-associated RCC, there have been attempts at mechanism-based treatment for HLRCC-associated RCC. FH-deficient kidney cancer is characterized by impaired Krebs cycle and oxidative phosphorylation, thus depends on glucose for adenosine triphosphate generation by aerobic glycolysis (Warburg effect). Increased oxidative stress and / or increased levels of fumarate inhibit hypoxia-inducible factor (HIF) prolyl hydroxylase which facilitates ubiquitinmediated degradation of HIF, resulting in HIF stabilization. Accumulation of HIF leads to increased transcription of vascular endothelial growth factor (VEGF) [2]. Bevacizumab can inhibit VEGF-mediated tumor vasculature. In a meanwhile, epidermal growth factor receptor (EGFR) signaling promotes aerobic glycolysis through the phosphoinositide 3-kinase / AKT or RAS / mitogen-activated protein kinase pathway [6], and EGFR TKI erlotinib reverted aerobic glycolysis in cancer cell line [7].

Against this backdrop, phase II clinical trials evaluating the efficacy and safety of the combination of bevacizumab plus erlotinib (AVATAR trial, NCT01130519) and the combination of vandetanib plus metformin (NCT02495103) are underway. An interim analysis of the AVATAR trial of bevacizumab plus erlotinib showed an impressive objective response rate (ORR) of $65 \%$ in patients with HLRCC-associated RCC [8] and a median progression-free survival (PFS) of 24.2 months. This promising result led to the recommendation of bevacizumab plus erlotinib for the treatment of HLRCCassociated RCC in the 2018 National Comprehensive Cancer Network (NCCN) guidelines [9].

In Korea, however, HLRCC-associated RCC has only recently been recognized by physicians and, to our knowledge, there are currently no formal reports on Korean patients with this disease. Therefore, the outcome of bevacizumab plus erlotinib therapy in Korean patients is not known. For this reason, we retrospectively collected data on patients with HLRCC in Korea and to evaluate the efficacy and safety of the bevacizumab plus erlotinib combination treatment.

\section{Materials and Methods}

\section{Patient selection}

We retrospectively reviewed consecutive patients diagnosed with HLRCC-associated RCC and treated between July 2014 and July 2018 in three academic hospitals (Asan Medical Center, Gil Medical Center, and Seoul National University Hospital) in South Korea. The selection criteria for analysis were as follows: diagnosis of HLRCC-associated RCC, advanced stage (recurrent, locoregionally advanced unresectable, or metastatic disease), treatment with bevacizumab (10 mg/ kg every 2 weeks intravenously) plus erlotinib (150 mg p.o daily) for HLRCC-associated RCC irrespective of line of treatment, and adequate clinical information and baseline and follow-up imaging.

Definitive diagnosis of HLRCC-associated RCC was confirmed by $\mathrm{FH}$ germline mutation test by polymerase chain reaction (PCR)-based sequencing methods from peripheral blood leukocytes. The criteria for proceeding to germline $\mathrm{FH}$ tests were as follows: suggestion of HLRCC-associated RCC in pathologic examination, diagnosis of papillary-type RCC under 40 years of age, pathologically confirmed cutaneous leiomyomatosis, multiple early-onset symptomatic uterine myoma in female patients or in family members of male patients, or family history of HLRCC syndrome.

\section{Data collection and analysis}

We collected the clinicopathological data of these patients from electronic medical record systems. Data on their baseline demographic characteristics, radiologic response and time-to-progression (TTP) to previous systemic treatments and bevacizumab plus erlotinib, adverse events related to bevacizumab plus erlotinib, and survival data were also gathered. Response evaluation according to Response Evaluation Criteria in Solid Tumors ver. 1.1 was performed by a comprehensive review of baseline and follow-up imaging. Usual radiographic evaluations were assessed every 2-3 months by computed tomography of the chest, abdomen, and pelvis but other image techniques were used at the discretion of the attending physicians. Safety was evaluated according to the Common Terminology Criteria for Adverse Events ver. 4.0, from the National Cancer Institute.

\section{Statistical analysis}

Patient characteristics and toxicity were summarized descriptively. The primary endpoint was ORR and the secondary endpoints were PFS, OS, and safety. Kaplan-Meier analysis was used to determine the time-to-event endpoints. PFS 
Table 1. Baseline patient characteristics

\begin{tabular}{|c|c|}
\hline Characteristic & $\begin{array}{c}\text { No. }(\%) \\
(n=10)\end{array}$ \\
\hline Age, median (range, yr) & $41(27-52)$ \\
\hline \multicolumn{2}{|l|}{ Sex } \\
\hline Male & $9(90)$ \\
\hline Female & $1(10)$ \\
\hline \multicolumn{2}{|c|}{ Tumor histology at the time of initial diagnosis } \\
\hline Papillary RCC & $8(80)$ \\
\hline Non-classified & $2(20)$ \\
\hline \multicolumn{2}{|c|}{ Disease status at the time of initial diagnosis } \\
\hline Localized & $3(30)$ \\
\hline Locally advanced & $2(20)$ \\
\hline Metastatic & $5(50)$ \\
\hline Nephrectomy & $6(60)$ \\
\hline \multicolumn{2}{|c|}{ Line of bevacizumab and erlotinib } \\
\hline 1 st & $4(40)$ \\
\hline 2nd & $1(10)$ \\
\hline 3rd or more & $5(50)$ \\
\hline \multicolumn{2}{|l|}{ IMDC prognostic group } \\
\hline Favorable & $1(10)$ \\
\hline Intermediate & $5(50)$ \\
\hline Poor & $4(40)$ \\
\hline \multicolumn{2}{|c|}{ Karnofsky performance status $(\%)$} \\
\hline $100-90$ & $2(20)$ \\
\hline $80-70$ & $5(50)$ \\
\hline$<70$ & $3(30)$ \\
\hline \multicolumn{2}{|l|}{ Metastatic sites } \\
\hline Lymph nodes & $9(90)$ \\
\hline Liver & $8(80)$ \\
\hline Bone & $7(70)$ \\
\hline Lung & $6(60)$ \\
\hline Adrenal gland & $3(30)$ \\
\hline \multicolumn{2}{|l|}{ No. of metastatic organs } \\
\hline 1 & 0 \\
\hline 2 & $3(30)$ \\
\hline 3 & $1(10)$ \\
\hline 4 or more & $6(60)$ \\
\hline
\end{tabular}

RCC, renal cell carcinoma; IMDC, International Metastatic Renal Cell Carcinoma Database Consortium.

was defined as the time from treatment initiation to the date of documentation of disease progression or death from any cause, whichever came first, while OS was calculated as the time from treatment initiation to death from any cause. Cases in which progression and/or death did not occur were censored at the date of the last follow-up. All statistical analyses were performed using IBM SPSS Statistics for Windows ver. 25.0 (IBM Corp., Armonk, NY).

\section{Ethical statement}

All procedures were conducted in accordance with the ethical standards of the institutional and/or national research committee and with the Helsinki declaration. This study was approved by the Institutional Review Board of each hospital (GAIRB2017-365). IRB waived informed consent for this study because of its nature of retrospective analysis.

\section{Results}

\section{Patient characteristics}

We identified 10 patients confirmed to have HLRCC-associated with RCC by FH germline mutation who received bevacizumab and erlotinib. Their basic demographic information is shown in Table 1 . The median age was 41 years (range, 27 to 52 years) and nine patients (90\%) were male. None were diagnosed with HLRCC-associated RCC by histologic examination at the time of initial diagnosis of RCC; that is to say, all cases of HLRCC-associated RCC were diagnosed by clinical suspicion and subsequent germline $\mathrm{FH}$ mutation testing. Seven patients (70\%) had locally advanced or metastatic disease at the time of initial diagnosis and radical nephrectomy was performed in six patients $(60 \%)$. The most common metastatic sites were lymph nodes, liver, and bone.

Five of the ten patients $(50 \%)$ received bevacizumab plus erlotinib as first- or second-line treatments, while five patients $(50 \%)$ received the combination as third-line or later. The majority of patients belonged to the intermediate or poorrisk groups according to the International Metastatic Renal Cell Carcinoma Database Consortium (IMDC).

\section{Systemic treatments for advanced disease}

Table 2 summarizes the history of systemic treatments and their outcomes. Among first-line treatments, five of the ten patients started with temsirolimus, one patient with sunitinib, and four patients with bevacizumab and erlotinib. Four patients treated with first-line temsirolimus showed no objective response, with a median TTP of 2.3 months. Four of them received axitinib as the second-line therapy, with ORR and TTP of 50\% and 9.2 months, respectively. One patient (case 1) achieved a long-term response with axitinib lasting about 25 months. Overall, VEGFR TKIs demonstrated favorable efficacy in a subset of patients, while mTOR inhibitors did not. Regarding immune checkpoint inhibitor therapy, three patients received ipilimumab plus nivolumab 


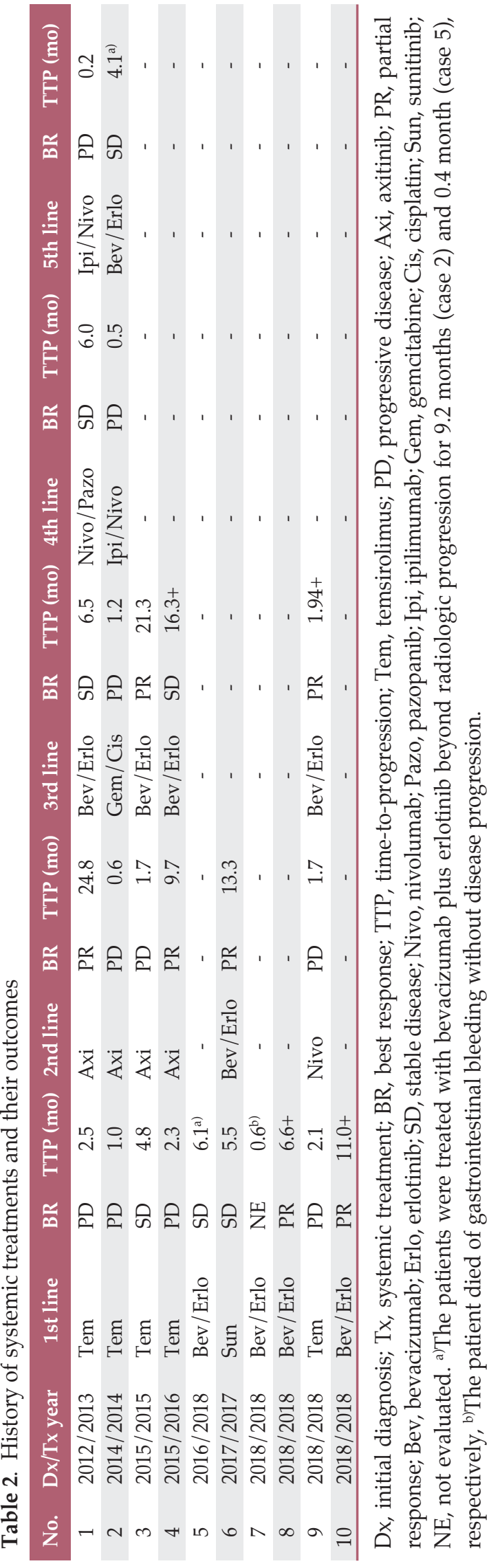

Table 3. Treatment efficacy of bevacizumab plus erlotinib in patients with HLRCC-associated RCC $(\mathrm{n}=10)$

\begin{tabular}{|c|c|}
\hline & Value \\
\hline \multicolumn{2}{|l|}{ Best overall response, $\mathrm{n}(\%)$} \\
\hline CR & 0 \\
\hline PR & $5(50)$ \\
\hline SD & $4(40)$ \\
\hline PD & 0 \\
\hline NE & $1(10)$ \\
\hline ORR $(95 \% \mathrm{CI}, \%)$ & $50(24-76)$ \\
\hline DCR $(95 \%$ CI, \%) & $90(60-98)$ \\
\hline Median PFS (95\% CI, mo) & $13.3(0.0-26.9)$ \\
\hline Median OS (mo) $)^{a}$ & 14.1 \\
\hline \multicolumn{2}{|c|}{$\begin{array}{l}\text { HLRCC, hereditary leiomyomatosis and renal cell carci- } \\
\text { noma syndrome; RCC, renal cell carcinoma; CR, complete } \\
\text { response; PR, partial response; } \mathrm{SD} \text {, stable disease; PD, pro- } \\
\text { gressive disease; NE, not evaluable; ORR, objective res- } \\
\text { ponse rate; CI, confidence interval; DCR, disease control } \\
\text { rate; PFS, progression-free survival; OS, overall survival. } \\
\text { a)The } 95 \% \text { CI was not calculated due to small number of } \\
\text { events. }\end{array}$} \\
\hline
\end{tabular}

(cases 1 and 2) or nivolumab (case 9) but they did not obtain any efficacy. Case 1 also received nivolumab plus pazopanib which were sustained for 6 months.

\section{Efficacy of bevacizumab and erlotinib}

For the patients who received bevacizumab plus erlotinib as second or more line of treatment, the median time from the first-line treatment to the initiation of bevacizumab plus erlotinib was 7.8 months. Among 10 included patients, nine patients underwent at least one response evaluation. Bevacizumab plus erlotinib resulted in a partial response in five patients $(50 \%)$, stable disease in four patients $(40 \%)$, and not evaluable in one (10\%), with the ORR of 50\% (95\% confidence interval [CI], 24 to 76) and disease control rate of 90\% (95\% CI, 60 to 98) (Table 3, Fig. 1). The estimated median PFS was 13.3 months (95\% CI, 0.0 to 26.9) (Fig. 2). Two patients (cases 2 and 5) continued bevacizumab plus erlotinib beyond radiologic disease progression at the discretion of the treating physicians because the speed of progression was slow, and the patients maintained good general conditions. Case 2 received bevacizumab plus erlotinib for an additional 9.2 months. The estimated median OS was 14.1 months (95\% CI, not calculated) (Fig. 2). 


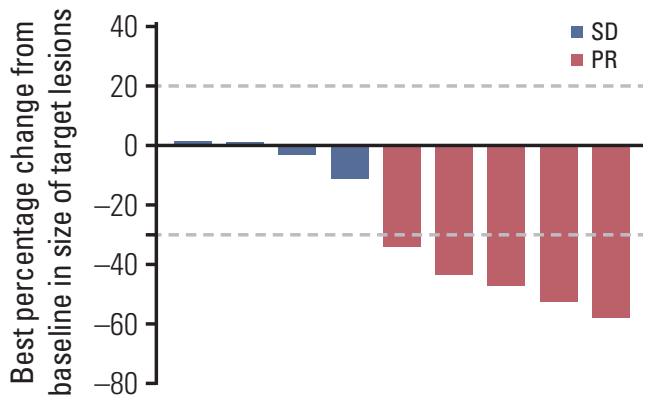

Fig. 1. Waterfall plots depicting change from baseline in sum of diameters for target lesions in patients with hereditary leiomyomatosis and renal cell carcinoma-associated renal cell carcinoma treated with bevacizumab plus erlotinib ( $\mathrm{n}=9)$. SD, stable disease; PR, partial response.

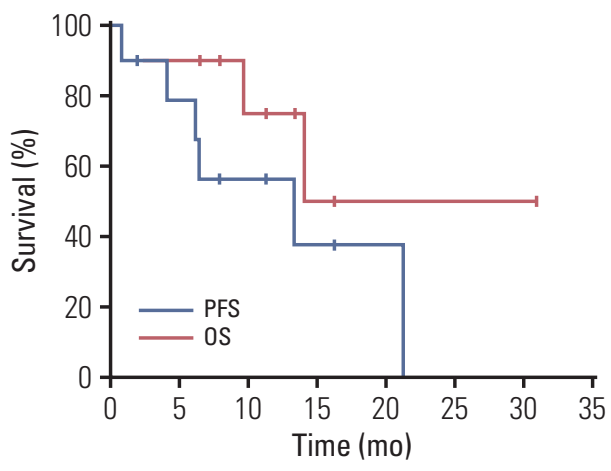

Fig. 2. Kaplan-Meier curve for progression-free survival (PFS) and overall survival (OS) in patient with hereditary leiomyomatosis and renal cell carcinoma-associated renal cell carcinoma treated with bevacizumab plus erlotinib.

Table 4. Adverse events of bevacizumab and erlotinib $(n=10)$

\begin{tabular}{|c|c|c|}
\hline & Any grade & Grade 3 or more \\
\hline Any adverse event & $10(100)$ & $2(20)$ \\
\hline \multicolumn{3}{|l|}{ Hematologic toxicity } \\
\hline Anemia & $2(20)$ & 0 \\
\hline Neutropenia & 0 & 0 \\
\hline Thrombocytopenia & 0 & 0 \\
\hline \multicolumn{3}{|l|}{ Non-hematologic toxicity } \\
\hline Acneiform eruption & $7(70)$ & $1(10)$ \\
\hline Hypertension & $5(50)$ & 0 \\
\hline Proteinuria & $4(40)$ & 0 \\
\hline ALT increased & $4(40)$ & 0 \\
\hline Dry skin & $4(40)$ & 0 \\
\hline Diarrhea & $3(30)$ & 0 \\
\hline Fatigue & $3(30)$ & 0 \\
\hline Creatinine increased & $3(30)$ & 0 \\
\hline Mucositis, oral & $2(20)$ & 0 \\
\hline Anorexia & $2(20)$ & 0 \\
\hline Insomnia & $2(20)$ & \\
\hline Hyperbilirubinemia & $1(10)$ & 0 \\
\hline Gastrointestinal bleeding & $1(10)$ & $1(10)^{a)}$ \\
\hline Dry eye & $1(10)$ & 0 \\
\hline Neuropathy & $1(10)$ & 0 \\
\hline Wound dehiscence & $1(10)$ & 0 \\
\hline Epistaxis & $1(10)$ & 0 \\
\hline Hand-foot syndrome & $1(10)$ & 0 \\
\hline
\end{tabular}

Values are presented as number (\%). ALT, alanine aminotransferase. ${ }^{a}$ The patient died of grade 5 gastrointestinal bleeding. 


\section{Safety profile of bevacizumab plus erlotinib}

All patients experienced any grade adverse events during treatment (Table 4). There were no unexpected toxicities. The most common event was acneiform eruption (70\%), followed by hypertension $(50 \%)$, proteinuria $(40 \%)$, alanine aminotransferase elevation (40\%), and dry skin $(40 \%)$. Grade 1 or 2 fatigue and diarrhea occurred one-third of the patients. These events were tolerable and managed with conventional supportive care. All patients continued treatment. However, one patient (case 7) died of gastrointestinal bleeding, which was not associated with disease progression but may have been an adverse event related to bevacizumab.

\section{Discussion}

To our knowledge, this is the first report of treatment outcomes not only in Korean patients diagnosed with HLRCCassociated RCC by germline genetic testing but also in realworld practice. We confirmed that the combination of bevacizumab and erlotinib is effective and safe. The ORR was 50\% and the median PFS and OS were 13.3 and 14.1 months, respectively. Adverse events were mostly predictable and manageable with routine supportive measures.

HLRCC-associated RCC was only recently categorized by $\mathrm{WHO}$ as a distinct entity; therefore, knowledge regarding its clinical features and prognosis is limited. In contrast to other hereditary RCC syndromes, HLRCC-associated RCC is usually unilateral and solitary and metastasis can occur even if primary lesion measures less than $1 \mathrm{~cm}[2,10]$. It usually develops in younger people, predominantly between the ages of 15 and 29 years, but can affect patients as young as 11 years [11]. In our study, the median age at diagnosis was 41 years (range, 27 to 52 years), similar to those in previous reports [12]. More than half of the patients in the present study had advanced disease at the time of diagnosis. The most common metastatic sites were lymph nodes (abdominal or thoracic), bone, and liver. None of our patients were suspected of having HLRCC-associated RCC by histologic examination. Clinical suspicion is important for a correct diagnosis; thus, physicians may be clued to suspicion by family history, presence of uterine myomas, skin lesions, and clinical course.

Early detection and prompt surgical extirpation can cure the condition, while the prognosis is very poor at advanced stages [13]. There has been no standard of care for metastatic HLRCC-associated RCC because definitive diagnosis is difficult and its rarity has precluded clinical trials. Thus far, AVATAR is the only clinical trial with promising results for the combination of bevacizumab plus erlotinib. In the interim analysis, the response rates were approximately $29 \%$ and $65 \%$ for papillary RCC without and with FH mutations, respectively. The median PFS in our group was shorter than that in a previous report (13.3 months vs. 24.2 months) [8], probably because patients with adverse features were included in our analysis. Furthermore, the small number and frequent censoring of the current study make the survival analysis less reliable.

All patients who received systemic treatment before 2018 started with mTOR inhibitor or VEGFR TKIs, according to clinical practice for non-clear cell RCCs. For these patients, bevacizumab plus erlotinib was administered in later lines. In contrast, four out of five patients diagnosed with metastatic disease in 2018 received bevacizumab and erlotinib as first-line treatment. The reason for the early initiation of bevacizumab plus erlotinib was the physician's awareness of this entity and guideline recommendation for this treatment. It is not clear whether patients treated with bevacizumab plus erlotinib as an initial treatment showed a better response rate and PFS than those in patients who had received other treatments before bevacizumab plus erlotinib. However, before other promising data are published, bevacizumab and erlotinib should be a standard treatment for HLRCC-associated RCC.

The adverse events of bevacizumab plus erlotinib treatment were mostly manageable. The most common adverse events were acneiform eruption, hypertension, proteinuria, liver function abnormality, and dry skin. Other constitutional symptoms such as fatigue, anorexia, or insomnia also occurred. One patient died of severe intestinal bleeding, which was deemed a complication of the bevacizumab. Therefore, although medical oncologists are familiar with the complications of both erlotinib and bevacizumab, care should be taken to detect potentially life-threatening complications.

In many cancers where targeted therapies like VEGF inhibitor or EGFR inhibitors are frequently used, adverse events attributable to the mechanism of action of molecular targeted agents have been increasingly suggested as predictive biomarker for efficacy $[14,15]$. We explored whether acneiform eruption, hypertension, or proteinuria could be a predictive marker for PFS. Median PFS was 6.5 (95\% CI, 0 to 15.5) and 13.4 (95\% CI, 0 to 27.4) for patients without and with acneiform eruption, respectively $(\mathrm{p}=0.36)$. In addition, median PFS was 6.2 months (95\% CI, 1.7 to 10.6) and 21.3 months (95\% CI, not calculated) for patients without and with hypertension ( $\mathrm{p}=0.023)$, and 6.2 months ( $95 \% \mathrm{CI}, 1.9$ to 10.5) and 21.3 months (95\% CI not calculated) for patients without and with proteinuria ( $\mathrm{p}=0.029)$ (S1 Fig.). To confirm the predictive role of these biomarkers, validation in larger homogeneous prospective cohort (like the AVATAR trial) is needed. 
Several prerequisites are essential to improving the clinical practice of HLRCC-associated RCC. Most importantly, physicians should be aware of HLRCC-associated RCC to properly diagnose and treat this disease. Although we reported patients with advanced-stage disease, it is important to detect and cure at early stages. Therefore, genetic counseling and screening of all family members are needed. Furthermore, active surveillance should be performed for early diagnosis of RCC in FH mutation carriers. This strategy has been suggested by the Kidney Cancer Research Network of Canada [16]. Additionally, the medical costs for genetic testing, surveillance, and treatment of HLRCC are currently not covered by the national health care insurance system in Korea. This financial burden might preclude families with FH mutations from proper and timely treatment and newlydetected families with this rare hereditary cancer syndrome comprise an urgent unmet medical needs in Korea.

This study has several limitations. Above all, this retrospective study included a small number of patients from three academic hospitals. Even though the three hospitals are among the largest in Korea, additional patients may have been diagnosed with HLRCC-associated RCC and received bevacizumab plus erlotinib. Second, there was no central pathology review. However, all participating centers were academic centers possessing genitourinary pathology specialists experienced in RCC. Finally, the PCR for FH was not centralized and was performed in each institution by laboratory-developed tests.

In conclusion, this is the first report on the treatment of advanced HLRCC-associated RCC in real-world practice. Bevacizumab plus erlotinib therapy was effective and the safety profiles were predictable and manageable. Physicians participating in the management of genitourinary cancer should be aware of HLRCC-associated RCC to properly diagnose and treat this disease. Considering its efficacy and safety, bevacizumab plus erlotinib should be the standard of treatment, unless other promising data are published.

\section{Electronic Supplementary Material}

Supplementary materials are available at Cancer Research and Treatment website (https: // www.e-crt.org).

\section{Conflicts of Interest}

Conflict of interest relevant to this article was not reported.

\section{References}

1. Tomlinson IP, Alam NA, Rowan AJ, Barclay E, Jaeger EE, Kelsell D, et al. Germline mutations in FH predispose to dominantly inherited uterine fibroids, skin leiomyomata and papillary renal cell cancer. Nat Genet. 2002;30:406-10.

2. Linehan WM, Rouault TA. Molecular pathways: Fumarate hydratase-deficient kidney cancer: targeting the Warburg effect in cancer. Clin Cancer Res. 2013;19:3345-52.

3. Lehtonen HJ. Hereditary leiomyomatosis and renal cell cancer: update on clinical and molecular characteristics. Fam Cancer. 2011;10:397-411.

4. Moch H, Cubilla AL, Humphrey PA, Reuter VE, Ulbright TM. The 2016 WHO classification of tumours of the urinary system and male genital organs-part A: renal, penile, and testicular tumours. Eur Urol. 2016;70:93-105.

5. Srinivasan R, Ricketts CJ, Sourbier C, Linehan WM. New strategies in renal cell carcinoma: targeting the genetic and metabolic basis of disease. Clin Cancer Res. 2015;21:10-7.

6. Yang L, Xie M, Yang M, Yu Y, Zhu S, Hou W, et al. PKM2 regulates the Warburg effect and promotes HMGB1 release in sepsis. Nat Commun. 2014;5:4436.

7. De Rosa V, Iommelli F, Monti M, Fonti R, Votta G, Stoppelli $\mathrm{MP}$, et al. Reversal of Warburg effect and reactivation of oxidative phosphorylation by differential inhibition of EGFR signaling pathways in non-small cell lung cancer. Clin Cancer
Res. 2015;21:5110-20.

8. Srinivasan R, Su D, Stamatakis L, Siddiqui MM, Singer E, Shuch B, et al. 5 Mechanism based targeted therapy for hereditary leiomyomatosis and renal cell cancer (HLRCC) and sporadic papillary renal cell carcinoma: interim results from a phase 2 study of bevacizumab and erlotinib. Eur J Cancer. 2014;50(Suppl 6):8.

9. National Comprehensive Cancer Network. NCCN clinical practice guidelines in oncology. Kidney cancer (ver 2. 2019) [Internet]. National Comprehensive Cancer Network; 2019 [cited 2019 Jan 3]. Available from: https:// www.nccn.org/professionals/physician_gls/pdf/kidney.pdf.

10. Grubb RL 3rd, Franks ME, Toro J, Middelton L, Choyke L, Fowler S, et al. Hereditary leiomyomatosis and renal cell cancer: a syndrome associated with an aggressive form of inherited renal cancer. J Urol. 2007;177:2074-9.

11. Alrashdi I, Levine S, Paterson J, Saxena R, Patel SR, Depani S, et al. Hereditary leiomyomatosis and renal cell carcinoma: very early diagnosis of renal cancer in a paediatric patient. Fam Cancer. 2010;9:239-43.

12. Merino MJ, Torres-Cabala C, Pinto P, Linehan WM. The morphologic spectrum of kidney tumors in hereditary leiomyomatosis and renal cell carcinoma (HLRCC) syndrome. Am J Surg Pathol. 2007;31:1578-85. 
13. Menko FH, Maher ER, Schmidt LS, Middelton LA, Aittomaki $\mathrm{K}$, Tomlinson I, et al. Hereditary leiomyomatosis and renal cell cancer (HLRCC): renal cancer risk, surveillance and treatment. Fam Cancer. 2014;13:637-44.

14. Dienstmann R, Brana I, Rodon J, Tabernero J. Toxicity as a biomarker of efficacy of molecular targeted therapies: focus on EGFR and VEGF inhibiting anticancer drugs. Oncologist. 2011;16:1729-40.
15. Sorich MJ, Rowland A, Kichenadasse G, Woodman RJ, Mangoni AA. Risk factors of proteinuria in renal cell carcinoma patients treated with VEGF inhibitors: a secondary analysis of pooled clinical trial data. Br J Cancer. 2016;114:1313-7.

16. Lattouf JB, Pautler SE, Reaume MN, Kim RH, Care M, Green J, et al. Structured assessment and followup for patients with hereditary kidney tumour syndromes. Can Urol Assoc J. 2016;10:E214-22. 